\title{
Metabolitos secundarios en Trichoderma spp. y sus aplicaciones biotecnológicas agrícolas
}

\section{Secondary metabolites in Trichoderma spp. and its agricultural biotechnological applications}

\author{
Ana María Mesa-Vanegas ${ }^{1^{*}}$, Alexander Marín², Jaime Calle-Osorno²
}

\begin{abstract}
Resumen
Trichoderma es un hongo con múltiples aplicaciones biotecnológicas agrícolas. La más importante es la capacidad de inhibir el crecimiento, la esporulación y la germinación de esporas de hongos patógenos. Una gran variedad de investigaciones se centra en estudiar el potencial biológico y la formulación a base de especies de Trichoderma con actividad inhibitoria sobre microorganismos, lo que genera que estas prácticas sean más eficaces en un amplio rango de condiciones ambientales, de especies de plagas y de sistemas de cultivos. La presente revisión ofrece una visión específica sobre los reportes de los metabolitos secundarios identificados en cepas del hongo de género Trichoderma con aplicación biotecnológica en la generación de productos agrícolas biocontroladores, con un enfoque prometedor para el descubrimiento de nuevos agentes de control basados en alternativas agroecológicas en el manejo integrado de hongos, bacterias, parásitos e insectos.
\end{abstract}

Palabras claves: agrobiotecnología, biocontroladores, hongos antagonistas, micoparasitismo

\begin{abstract}
Trichoderma is a fungus with multiple agricultural biotechnological applications. The most important is its ability to inhibit the growth, sporulation and germination of spores from pathogenic fungi. A wide variety of research focuses on studying the biological potential and formulation based on Trichoderma spp. with inhibitory activity on microorganisms; the result of these research makes the practices in which the fungus is used more effective in a wide range of environmental conditions, species of pests and crop systems. This review offers a specific view on reports of secondary metabolites identified in strains of fungi of the genus Trichoderma with biotechnological application in the generation of biocontrol agricultural products, with a promising approach for the discovery of new control agents based on agroecological alternatives in the integrated management of fungi, bacteria, parasites and insects.
\end{abstract}

Keywords: agrobiotechnology, biocontrol, antagonist fungi, mycoparasitism

\footnotetext{
1. Grupo de investigación Agrobiotecnología, Universidad de Antioquia, Instituto de Biología, Medellín, Colombia.

2. Grupo de investigación BIOMA, Universidad de Antioquia, Instituto de Biología, Medellín, Colombia.

* Autor de correspondencia: <amaria.mesa@udea.edu.co>
} 


\section{INTRODUCCIÓN}

Durante los últimos años los sistemas agrícolas se han visto afectados por el uso de agroquímicos para controlar el ataque de malezas, plagas y enfermedades, lo que ha generado graves problemas de salud animal y de carácter medioambiental. Entre estos problemas se cita la reducción de la biodiversidad como uno de los más importantes, seguido de la pérdida en la salud del suelo (FAO 2019, Ruane y Sonnino 2011). La agricultura convencional es cada vez menos sostenible debido a la presión ejercida por otro tipo de prácticas y manejos con enfoques sostenibles, mercados diferenciados y exigentes; los cuales, a través de organismos reguladores, hacen cumplir estándares de producción específicos, además de que existe un creciente problema de resistencia a los plaguicidas que está forzando a la industria a buscar alternativas diferentes de manejo (Gavrilescu y Chisti 2005, Saedd et al. 2017).

La biotecnología agrícola ha beneficiado a agricultores, consumidores y al medio ambiente, dado que los rendimientos y los ingresos agrícolas de productos biotecnológicos han aumentado, disminuyendo las aplicaciones de pesticidas y mejorando el suelo, la calidad del agua y proporcionando alimentos saludables para los consumidores (Gavrilescu y Chisti 2005, Pathak et al. 2017). La investigación sobre productos biológicos plaguicidas se ha fortalecido debido al incremento en el resurgimiento de plagas, los efectos negativos de los plaguicidas sobre el medio ambiente y la biodiversidad y una mayor demanda de alimentos seguros por parte de los consumidores (Adekambi et al. 2010, Cantrell et al. 2012).

Dentro de las líneas de interés en el estudio de agentes controladores de plagas se encuentran aquellos basados en hongos del género Trichoderma; estos organismos son de suma importancia para la comunidad científica gracias a sus propiedades celulolíticas, lo que ha permitido desarrollar herramientas moleculares para su manipulación, con la consecuente secuenciación del genoma del aislado original Trichoderma reesei QM6a, también conocido como Hypocrea jecorina (Zeilinger et al. 2016). Varios de sus mutantes son productores de celulasas y son ampliamente usados en la industria. El genoma haploide de T. reesei QM6a se estimó en $33 \mathrm{Mb}$, organizado en siete cromosomas. En la actualidad existen dos bancos de información especializado en Trichoderma, NCBI y ISTH.info, el cual es un sitio web oficial de la Subcomisión Internacional sobre Taxonomía de Trichoderma e Hypocrea (http://www.isth.info/, Mukherjee et al. 2012, Zeilinger et al. 2016).

El éxito de estos organismos en la agricultura se centra en sus habilidades micoparásitas a través de mecanismos de competencia por espacio, nutrientes y producción de compuestos antimicrobianos que actúan sobre organismos patógenos de plantas (Harman y Kubicek 2014). Esta habilidad para el control biológico se debe a la profunda capacidad de Trichoderma de expresar metabolitos que parasitan o atacan otros organismos; al punto tal, que hoy en día esta propiedad se reconoce como la naturaleza innata de todo el género. Esta propiedad ha permitido su comercialización a pesar de que existen pocos bioplaguicidas de éste tipo registrados en el mercado. En su mayoría, los productos comerciales del mercado se formulan con una concentración de antagonista entre $1 \times 10^{8-9}$ u.f.c esporas viables / gramo de producto formulado (Gupta et al. 2014).

Actualmente, Trichoderma ofrece mucho más a la ciencia al poderse aislar de una innumerable diversidad de sustratos naturales y artificiales, y particularmente de materiales infestados con xenobióticos, demostrando su alto potencial oportunista y adaptabilidad a varios sistemas ecológicos (HoyosCarvajal 2012, Martínez et al. 2013). Como asociados asintomáticos de plantas (endófitos), estos hongos las benefician mediante la supresión de patógenos, la promoción del crecimiento, la mejora en la toma y disponibilidad de nutrientes y la disponibilidad e inducción de resistencia. Todas estas propiedades hacen que Trichoderma sea uno de los géneros fúngicos más versátiles e intrigantes, con numerosos aspectos para tratar con más detalle (Benitez et al. 2004, Gakuya et al. 2013).

El estudio de la diversidad y las interacciones estructurales y funcionales dentro de la comunidad de Trichoderma, son cuestiones esenciales para comprender los ecosistemas microbianos (Hoyos-Carvajal 2012). En la actualidad existe una tendencia al uso 
de estos organismos en el control biológico como antagonista sobre distintos patógenos de suelo por la producción prolífica de metabolitos secundarios MS (Sivasithamparam y Ghisalberti 1998, Zeilinger et al 2016). El género presenta un amplio espectro de aplicación agrícola sobre bacterias, parásitos e insectos, pero son pocos los estudios basados en la producción de sus metabolitos activos como una base para su explotación biotecnológica (Harman y Kubicek 2014). Debido a lo anterior, la Agencia de Protección Ambiental de los Estados Unidos (EPA) tiene en sus registros información donde se demuestra que no solo el organismo biológico es empleado para el control, sino también los extractos, aceites esenciales y MS obtenidos a partir de este, que juegan un papel importante en el descubrimiento y desarrollo de nuevos productos para la protección de cultivos bajo el concepto del Manejo Integrado de Plagas y Enfermedades (IPDM) (Cantrell et al. 2012, EPA 2016).

El presente trabajo tiene como objetivo una revisión en bases de datos (Science Direct, PUBMED, Scielo en enero 2019), libros especializados, referencias bibliográficas, artículos científicos y revistas indexadas, entre el 2005 y 2018, relacionados con la utilización de especies de Trichoderma spp., como un microorganismo benéfico en la producción agrícola, sus interacciones bioquímicas, moleculares y específicamente en la expresión de los principales MS. Además, se presenta y se discuten los métodos experimentales para la obtención, identificación y caracterización de MS reportados en Trichoderma, así como los métodos para determinar la actividad de los MS mediante macro y micro métodos sobre hongos, bacterias, parásitos e insectos plaga en plantas con su aplicación biotecnológica.

\section{BIOLOGÍA DE Trichoderma spp.}

\section{Características macro y microscópicas}

Los hongos filamentosos del género Trichoderma (pertenecientes a la familia Hypocreaceae), son cosmopolitas en suelo, madera y hortalizas en descomposición, muestran una notable gama de estilos de vida e interacciones con otros hongos, animales o plantas, lo que les confiere alta plasticidad ecológica, capacidad enzimática para degradar sustratos, metabolismo variable y resistencia a inhibidores microbianos (Lorito 2006). Estos hongos son ascomicetos de aspersión verde, con distribución mundial, que en su mayoría no se han asociado con un estado sexual y se cree que son mitóticos y clonales (Gupta et al. 2014). Los géneros en el orden de los Hypocreales tienen anamorfos referibles a Trichoderma; en los últimos años se ha vinculado un número creciente de teleomorfos en Hypocrea.

En Trichoderma, la taxonomía es aún bastante incompleta y la distinción de especies es compleja; otros métodos taxonómicos suplementarios a la morfología incluyen estudios moleculares como caracterización de proteínas o perfiles de isoenzimas, secuencias de la región ITS 1 y 2 del ADN ribosomal, genes tef1, rpb2, cal1, chi18-5 y técnicas de fingerprinting. Además del estudio de los MS, que muestran una gran diversidad en este género y que han permitido en los últimos años una mejor resolución de las entidades taxonómicas (Reino et al. 2007), es necesaria una clasificación e identificación refinada para las indicaciones predictivas sobre la ecología, toxicología y biotecnología de este género (Contreras-Cornejo et al. 2016).

Morfológicamente se caracteriza por presentar un ralo fino, los conidióforos son ramificados como un árbol pequeño, penachos compactados en forma de anillo con sistema de ramas irregular de manera piramidal, los cuales terminan en fiálides en forma de esporas asexuales o conidios, que pueden emerger directamente del micelio. Clamisdosporas intercalares, terminales y propágulos de tres tipos: hifas, clamidosporas y conidios. Igualmente, como método de resistencia, los hongos de este género tienen la capacidad de producir clamidiosporas, las cuales pueden producirse en zonas sociales, es decir, en zonas terminales del micelio, como en zonas medias o intercalados (figura 凹).

\section{Cultivo}

Al ser Trichoderma un género cosmopolita y compartir una amplia variedad de interacciones con diferentes organismos, es fácilmente aislado del suelo por métodos convencionales, en gran parte debido a su rápido crecimiento, abundante conidiación, for- 


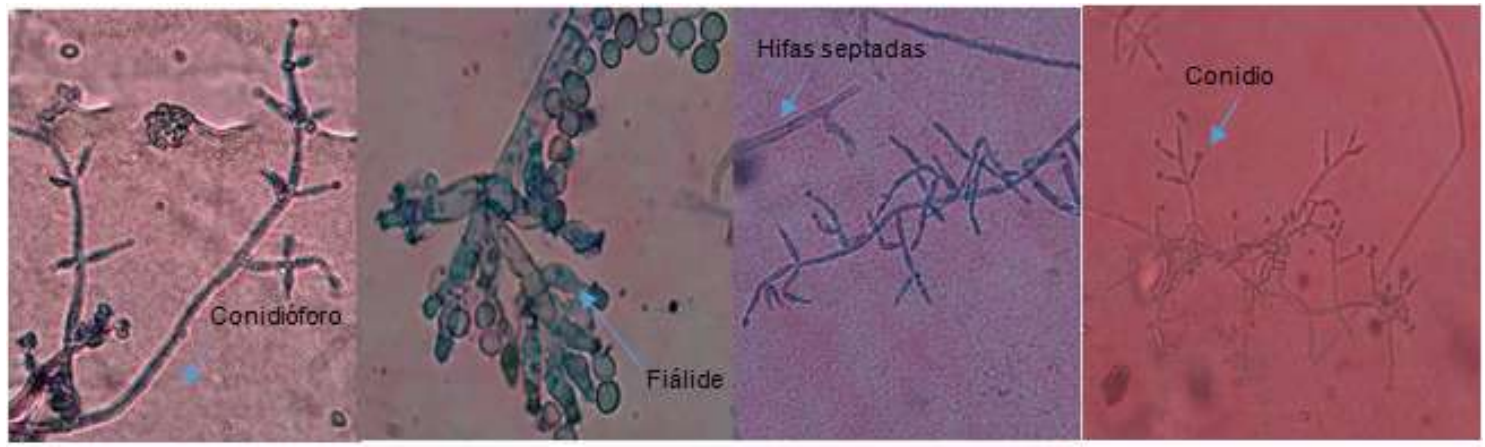

Figura 1. Fotografías tomadas al microscópico óptico de cepas del género Trichoderma spp. conservadas en el laboratorio de Biocontrol y Microbiología Ambiental del Instituto de Biología de la Universidad de Antioquia (BIOMA).

mación de clamidiosporas y colonización de sustratos orgánicos, lo que facilita un rápido desarrollo en varios sustratos (Gupta et al. 2014). Para el mantenimiento y la conservación de estos hongos se emplea el medio de cultivo: agar extracto de malta (MEA), en la oscuridad por dos días a temperatura ambiente. Para el crecimiento lineal, incubación en la oscuridad durante cuatro días a $20 \pm 1^{\circ} \mathrm{C}$ y para la inoculación de conidias, se emplea medio diluido al $2 \%$ de agar MEA. Las tasas de crecimiento se determinan en los medios de cultivo agar bajo en nutrientes (SNA) y agar papa dextrosa (PDA). La observación microscópica en los cultivos permite diferenciar agregados conidiales y pigmentos en el sustrato característicos de algunas especies (Eziashi et al. 2006). La morfología del conidióforo y el patrón de ramificación, crítico para la identificación a nivel seccional, se observan mejor antes que los conidios estén completamente maduros. El material se monta en ácido láctico y con la ayuda de aceite de inmersión se pueden establecer detalles de forma conidial y ornamentación. Las tasas de crecimiento en cultivo pueden ser útiles para diferenciar las especies, considerando que la producción de conidios a partir de conidióforos o de conidióforos agregados en fascículos o pústulas es generalmente característico de una especie (Harman y Kubicek 2014).

La expresión de MS mediante la generación de pigmentos difusibles también puede ser característico de una especie, aunque el color de dichos pigmentos no varía mucho en Trichoderma (Schulz et al. 2002). La mayoría en su inicio presentan color blanco, que se torna a verde o amarillento con esporulación densa; algunas cepas referibles a Longibrachiatum suelen tener pigmentos brillantes de color amarillo verdoso brillante. Los pigmentos amarillentos apagados a varios tonos verdes y grises son comunes en muchas especies, pero no son muy distintivos (Gupta et al. 2014). En algunas especies, los conidios maduros aparecen de color verde oscuro en el microscópico, otras especies se caracterizan mejor por una completa falta de pigmento, mientras que los pigmentos rojizos se producen en unos pocos aislamientos característicos en T. aureoviride. Los olores aromáticos que se asemejan al coco son producidos comúnmente por cepas de $T$. viride y algunas veces también por $T$. atroviride (Harman y Kubicek 2014) (figura ㄱ) .

La distribución, filogenia, ecología, mecanismos de defensa e interacción deletérea con huéspedes, producción y secreción de enzimas, desarrollo sexual y respuesta al medio ambiente en condiciones tales como nutrientes y luz, son tópicos que han sido ampliamente estudiados en este género (Vinale et al. 2008, 2012). Las cepas de Trichoderma pueden ejercer el biocontrol de hongos fitopatógenos indirectamente, compitiendo por espacio y nutrientes, modificando las condiciones ambientales, estimulando el crecimiento de las plantas y sus mecanismos de defensa o mediante la producción de compuestos antimicrobianos. También pueden realizar ese biocontrol directamente, mediante micoparasitismo.

Estos mecanismos pueden actuar de forma coordinada y su importancia en los procesos de biocontrol 

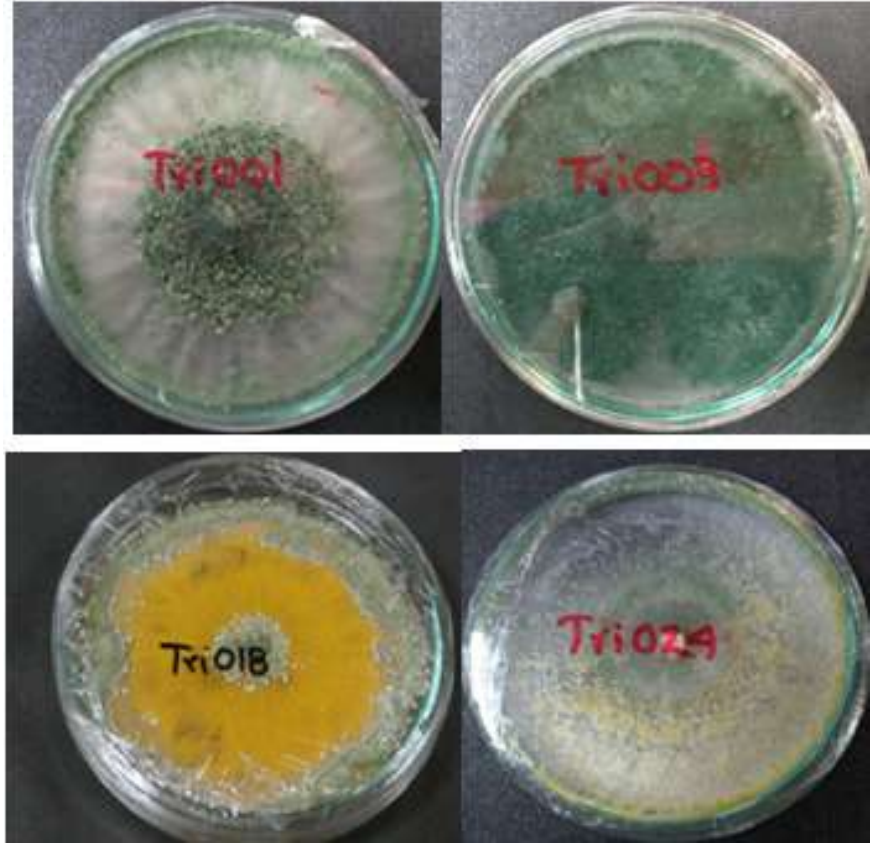

Figura 2. Fotografía de la apariencia de las colonias de diferentes cepas del género Trichoderma spp. aisladas y conservadas en cultivo PDA en el laboratorio de Biocontrol y Microbiología Ambiental del Instituto de Biología de la Universidad de Antioquia (BIOMA).

depende del hongo y del patógeno al que antagoniza, del tipo de cultivo y de condiciones ambientales como la disponibilidad de nutrientes, el $\mathrm{pH}$, la temperatura o la concentración de hierro (Harman y Kubicek 2014).

Entre las aplicaciones agrícolas se han registrado metabolitos que inhiben directamente a patógenos, aumentan la resistencia a enfermedades y mejoran el crecimiento y producción de las plantas. Su mecanismo de acción biocontroladora fue descrito ampliamente y está basado en el micoparasitismo, la antibiosis, la competencia por espacio y nutrientes y la acción directa sobre el patógeno (Zeilinger et al. 2016). Estos mecanismos se han propuesto dada la expresión de enzimas y MS que actúan mediante distintos mecanismos como inhibidores de crecimiento, elicitores o inductores de mecanismos fisiológicos en la planta y detoxificación de toxinas excretadas por patógenos, entre otros.

Los MS son cruciales para el establecimiento de interacciones entre plantas y microrganismos, como en el caso de las interacciones Trichoderma-planta (Verma et al. 2007). Sus características controladoras como fungicidas, antibióticas, nematicidas e insecticidas y sus aplicaciones como colonizadores de sus hábitats, han permitido la comprensión en cuanto a la producción de potentes MS como agentes descomponedores del suelo con funciones específicas. La preservación de Trichoderma es importante porque asegura la viabilidad e integridad morfológica, fisiológica y genética de un cultivo para su utilización en la producción del hongo o de sus metabolitos manteniendo las características particulares de la cepa original (Vinale et al. 2012).

\section{METABOLISMO SECUNDARIO EN Trichoderma spp.}

Las características básicas de la producción de energía y el crecimiento celular en todos los organismos son esencialmente similares y las moléculas implicadas en las reacciones fundamentales del crecimiento y la reproducción son las mismas. Estas biomoléculas ubicuas y esenciales son aminoácidos, carbohidratos, ácidos grasos y ácidos nucleicos; se clasifican como metabolitos primarios (MP) y participan como factores de crecimiento en plantas o como enzimas líticas, sideróforos, antibióticos, permeasas de carbono y nitrógeno. Otra característica de los MP es que sus concentraciones en las células son controladas. Por su parte, los MS son ampliamente divisibles en varias agrupaciones características como policétidos, terpenos, terpenoides, pirógenos, fenoles y alcaloides derivados de compuestos indólicos y péptidos no ribosómicos, que en general reflejan su origen y biosíntesis. Las diferentes especies de una misma familia y diferentes aislamientos de una misma especie, a menudo pueden producir MS significativamente diferentes que conllevan a la individualidad de las especies (Gupta et al. 2014, Harman y Kubicek 2014). Dado que estos hongos son habitantes comunes del suelo y de la raíz de las plantas, diversos MS se han aislado, caracterizado y han sido ampliamente estudiados debido a su actividad como antimicrobianos. La activación de cada uno de los mecanismos bioquímicos implicados en la producción y diversidad de los MS son la base de estudio en la diversidad de 
especies en el género Trichoderma (Keswani et al. 2014).

En cuanto a la expresión de los MS, esta depende de las características genéticas y ambientales de los hongos. Las bondades como agente de control dependen más de las cepas de Trichoderma que de la especie, pues estas pueden presentar diferencias en sus modos de acción, aún perteneciendo a una misma especie. Uno de los primeros estudios de metabolitos aislados del genero Trichoderma, fue realizado por Weindling en 1932 donde obtuvo un compuesto cristalino antibiótico de T. lignorum UAMH 5068 antagónico a otros hongos (Andrade et al. 1992); dicho compuesto fue denominado gliotoxina junto con otro compuesto aislado denominado viridino que exhibieron actividad fungicida (figura 3 ).

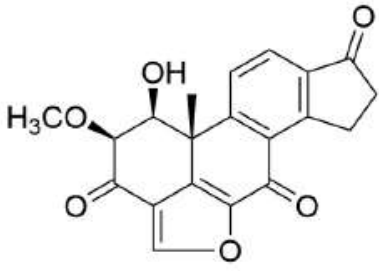

Viridino

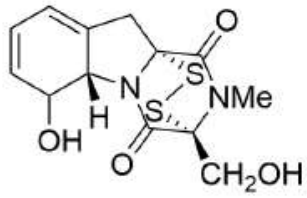

Gliotoxina
Figura 3. Primeros metabolitos aislados de Trichoderma spp.

Otra especie que ha sido estudiada, mediante un análisis químico, es T. hypoxylon CGMCC 3.17906, en la cual se observó una riqueza de metabolitos tipo tricotecenos y epipolitiodicetopiperazinas donde los principales compuestos aislados y caracterizados fueron Harzianum B, Aspergillazina C, Aspergillazina A, Trichodermamida A, con una versatilidad molecular que los hace bastante interesantes en la comprensión del estilo de vida del hongo y el rol ecológico de T. hypoxylon en la naturaleza (figura 国). Estos metabolitos pueden sobre expresarse o combinarse con cepas de biocontrol apropiadas, a fin de obtener nuevas formulaciones que puedan ser más eficaces en el control de enfermedades de plantas y en la protección de frutos post-cosecha (Sun et al. 2016).

\section{ACTIVIDAD ANTIFÚNGICA DE METABOLITOS DE Trichoderma}

Un extracto de Trichoderma contiene una mezcla de compuestos que puede ser utilizada para control biológico y otros fines industriales (figura (19) (Marques et al. 2018). Se ha comprobado el efecto antifúngico de la cepa T. atroviride S361 en cultivo sólido de arroz y del cual se han aislado tres nuevos compuestos denominados trichodermonas A-C. Igualmente, de esta misma cepa se aislaron (-)trichodermadiona A, $(+)$ trichodermadiona A, y un nuevo sesquiterpenoide ciclohexenona denominado trichodermadiona B. El efecto de estas cepas y sus MS fueron evaluados en hongos fitopatógenos con promisoria actividad (Kong et al. 2018, Zhou et al. 2017). Por otra parte, dos cepas de Trichoderma fueron empleadas en cultivos de Vitis vinifera presentado efectos de inducción de resistencia a enfermedades, promoción del crecimiento de las plantas y aumento de polifenoles o actividad antioxidante en las uvas (Pascale et al. 2017). Las cepas de T. harzianum M10 o T. atroviride $\mathrm{P} 1$, y sus $\mathrm{MS}$, ácido harziánico (HA) y 6-pentil-a-pirona (6PP), se han evaluado en invernadero mediante aplicación foliar. A partir de estos compuestos se desarrolló un fungicida biológico a base del hongo $T$. harzianum $\left(\mathrm{T}_{12}\right)$ para el control de varias enfermedades fungosas del suelo que afectan cultivos agrícolas en Mérida, Venezuela. La formulación se obtuvo a partir de una cepa aislada de un suelo de tradición ajera proveniente del municipio de Rivas Dávila, donde la cepa mostró, en pruebas de laboratorio y de campo, una alta capacidad antagónica contra los hongos Rhizoctonia solani, Sclerotium cepivorum, Sclerotium rolfsii, Fusarium sp., Plasmodiophora brassicae y Phytohthora sp., logrando reducciones de la incidencia de éstas enfermedades superiores a $25 \%$, en dependencia de ellas y de las condiciones ambientales (García et al. 2006). En un experimento en campo, se aplicó una suspensión de esporas de T. harzianum cepa T22 y 6PP; y los resultados indicaron que ambas cepas son capaces de mejorar el rendimiento del cultivo y aumentar la cantidad total de polifenoles y la actividad antioxidante en las uvas (Mona et al. 2017). Otros estudios registran también la validación con el uso de este antagonista para el manejo de Rhizoctonia solani, evidenciando un control de la enfermedad que puede 


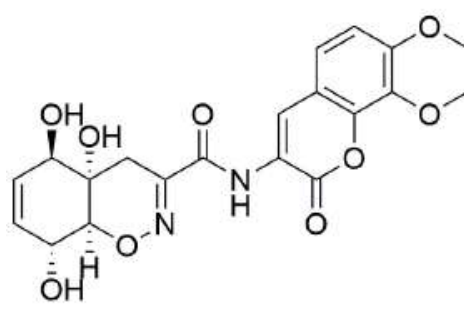

Trichodermamida A

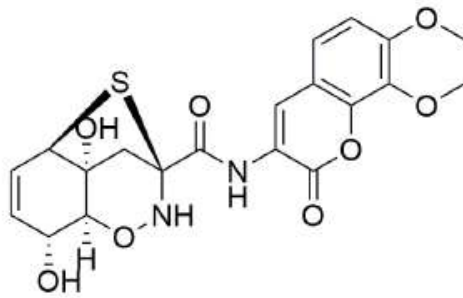

Aspergilazina A

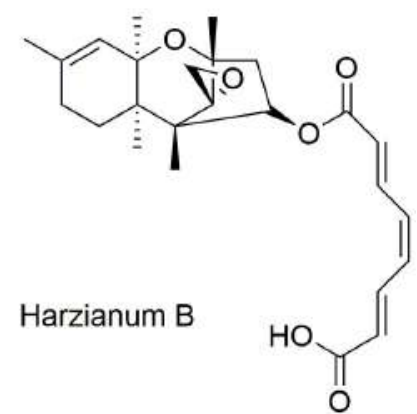

Figura 4. Compuestos aislados y caracterizados de T. hypoxylon.

llegar hasta $98 \%$ (Zhang et al. 2016). Esta cepa se ha probado con éxito en cultivos de papa y otras solanáceas, también en ajo, crucíferas, leguminosas, plátano, café y tabaco, entre otros (Saleh et al. 2018). Por otra parte, se extrajeron MS de 128 aislados de Trichoderma de cultivos líquidos, donde se evaluó su actividad frente a siete aislamientos de Phytophthora. Luego del análisis preliminar, se seleccionaron ocho aislamientos de Trichoderma para pruebas adicionales. Entre estos, los metabolitos de T. atroviride / petersenii (KACC, Colección de Cultivos de Corea, 40557) y T. virens (KACC 40929) mostraron las actividades inhibitorias más fuertes contra aislados de Phytophthora. El tratamiento con KACC 40557 inhibió el crecimiento de Phytophthora e indujo genes relacionados con la defensa, además provocó cambios hormonales en la planta durante la infección por Phytophthora y en las hojas desprendidas de las plantas de pimienta y tomate, lo que demostró el potencial para el uso de metabolitos de Trichoderma como agentes de control biológico contra este hongo patógeno (Bae et al. 2016). La interacción Trichoderma-Trichoderma de 35 cepas, fue estudiada tambien debido al potencial del hongo para sintetizar MS, mediante enfrentamientos duales en medio de cultivo sólido PDA como método de selección para cepas formadoras de barreras de defensa entre 0,5-0,7 cm con ancho óptimo de selección. Se identificaron 20 enfrentamientos duales, los cuales fueron replicados en medio de cultivo líquido de PDA y filtrados para separar los MS (Puño et al. 2011). Este compuesto de interés, que contiene los MS, fue inoculado en dos cultivos: Lactuca sativa y Raphanus sativus; encontrándose que los mejores tratamientos para el ensayo de
L. sativa fueron los enfrentamientos de TrichodermaTrichoderma 3(BP-T0001-BPT0029), 4(BPT0006BPT0028), 7(BP-T0007-BPT0024) y del cultivo individual I (BP-T0028) induciendo el crecimiento en la planta y la resistencia al ataque de hongos. Por lo que se concluyó que los MS sintetizados por Trichoderma spp. durante los enfrentamientos duales actúan como Promotores del Crecimiento en Plantas (PGP) y en la resistencia al ataque de hongos fitopatógenos (Okuda et al. 1982).

También se ha estudiado el efecto inhibidor in vitro del desarrollo de Alternaria citri, Bipolaris cynodontis, Bipolaris sorokiniana, Curvularia brachyspora, Curvularia lunata, Curvularia oryzae-sativae, Drechslera tritici-repentis, Rhizoctonia solani, Sclerotinia minor y Sclerotium rolfsii, causado por los componentes volátiles como etileno producidos por T. hamatum (Ghisalberti y Rowland 1993, León et al. 2017). Los organismos se cultivaron y se determinó, por cromatografía de gases acoplada a espectrometría de masa (GC-MS), la variación de dióxido de carbono, oxígeno y etileno, sin encontrarse rastros de acetaldehído ni etanol. Debido a la actividad respiratoria de $T$. hamatum, los niveles de dióxido de carbono aumentaron progresivamente, mientras disminuía el volumen de oxígeno. La producción de etileno fue baja y después del tercer día permaneció constante. Con excepción de $C$. oryzae-sativae y $B$. cynodontis, las demás especies fúngicas presentaron alteraciones en su crecimiento y desarrollo, concluyendo que $T$. hamatum actúa como potencial agente de control biológico mediante la expresión de sus MS 


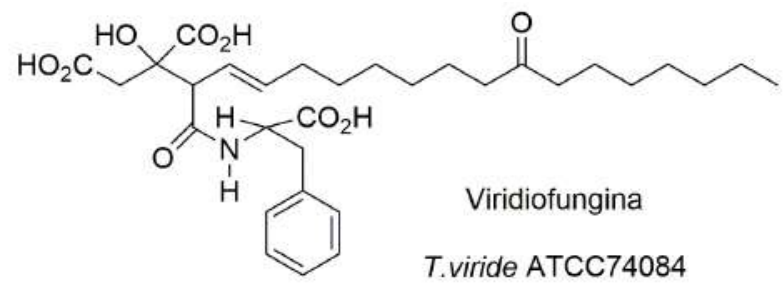<smiles>C/C=C/CC(C)C(=O)C1=C(C)NC(OC)C(OC)=C1O</smiles>

T. harzianum IMI298371<smiles>CCC/C=C/c1cccc(=O)o1</smiles>

6-pent-1-enil- $\alpha$-pirona

T. harzianum IMI275950

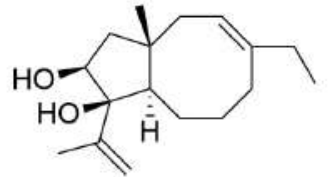

3,4-dihidroxicarotano

T.virens ATCC74180<smiles>CC=CC=CCC1=C(CC(C)O)C(=O)OC1</smiles>

T. harzianum IMI311092

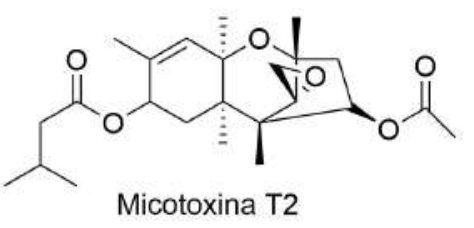

T.lignorum<smiles>COc1ccc2cc(NC(=O)C3=NO[C@@H]4[C@@H](O)C=C[C@H](O)[C@]4(O)C3)c(=O)oc2c1OC</smiles>

Trichodermamida A

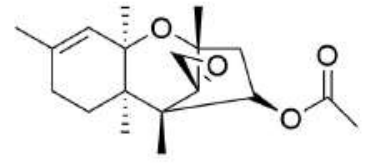

Tricodermina

T.polyspoum CMI 40624

T.sporulosum CMI104643
Harzianum A

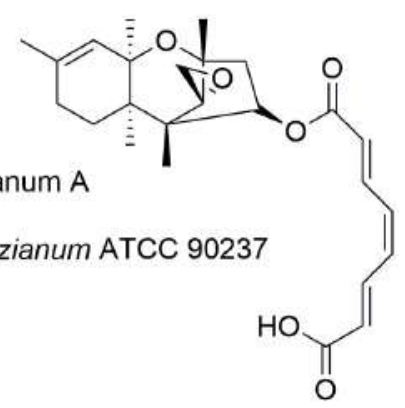<smiles>COc1ccc2cc(NC(=O)C34CC5(O)CC36C(ON4)[C@@H](O)C=CC56)c(=O)oc2c1OC</smiles><smiles>CC(C)[C@H](C)/C=C/[C@H](C)[C@H]1CCC2C3=CC=C4C[C@@H](O)CC[C@]4(C)C3CC[C@]21C</smiles>

Aspergilazina A

Figura 5. Diversidad de metabolitos aislados de Trichoderma spp. 
(Dal Bello et al. 1997).

Yang et al. (2012) emplearon la técnica de extracción Headspace para evaluar la actividad debida únicamente a compuestos orgánicos volátiles (COVs). Los resultados indican que los COVs liberados por $T$. viride afectan el crecimiento de los hongos fitopatógenos evaluados. En el caso de Fusarium sp. se afectaron los halos de crecimiento y para Colletotrichum gloeosporioides se observaron cambios morfológicos en su color. Se identificaron mediante técnicas de extracción e instrumentales (GC-MS) alcoholes,

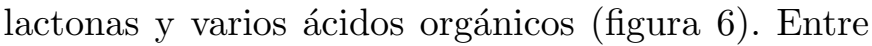
los compuestos determinados por las tres técnicas se encuentra el alcohol bencílico, alcohol 2-feniletílico, 6-pentil-2H-piran-2-ona y gama-butirolactona (León et al. 2017, Yang et al. 2012). Por otra parte, se ha incorporado T. harzianum en cultivos de arroz con una reducción de hasta $50 \%$ de los esclerocios y de $13,4 \%$ en la severidad de $R$. solani (Infante et al. 2009).

\section{Actividad antibacterial de metabolitos de Tricho- derma}

Un amplio repertorio de metabolitos biológicamente activos es utilizado por especies de Trichoderma para la exclusión de patógenos, incluida la competencia por espacio, nutrientes y producción de compuestos antibacterianos (figura (5) (Smirnova et al. 2017). En la búsqueda de productos con aplicaciones antimicro- bianas, un total de 49 cepas fúngicas fueron aisladas de dos cenotes de la península de Yucatán. Estas cepas se cultivaron en arroz fermentado y se obtuvieron sus respectivos extractos orgánicos macerados en acetato de etilo y metanol y se evaluaron contra cinco fitopatógenos de importancia agrícola. Estos patógenos incluyeron a los hongos Alternaria chrysanthemi, Colletotrichum gloeosporioides, Mycosphaerella fijiensis y las bacterias Erwinia carotovora y eXanthomonas campestris. Utilizando el bioensayo en microdilución, el $69 \%$ de los extractos evaluados mostró actividad antifúngica y/o antibacteriana contra al menos uno de los patógenos evaluados a concentraciones de 2.000 y $200 \mu \mathrm{g} / \mathrm{mL}$ (Elfita et al. 2014). Por otra parte, la cepa Hypocrea lixii OSN-37 ( Trichoderma) mostró actividad contra al menos tres bacterias fitopatógenas. Los extractos de acetato de etilo fueron los más promisorios obteniendo tres fracciones de baja (A) y media (B) polaridad y un precipitado (C), los cuales fueron evaluados contra los patógenos estudiados (Saravanakumar et al. 2018). Dos nuevos derivados de policétidos con actividad antibacterial, trichodermatidas $\mathrm{E}$ y $\mathrm{F}$ con esqueleto tipo 6/6/6/6 tetracíclicos y análogos conocidos como koninginina $\mathrm{B}$, koninginina $\mathrm{D}$, 7-O-metilkoninginina $\mathrm{D}$, koningininas $\mathrm{E}$ y $\mathrm{F}$, fueron aislados del hongo $T$. applanatu. Las estructuras de estos compuestos se determinaron mediante datos de resonancia magnética nuclear y análisis de espectrometría de masas de alta resolución con ionización de tipo electrospray
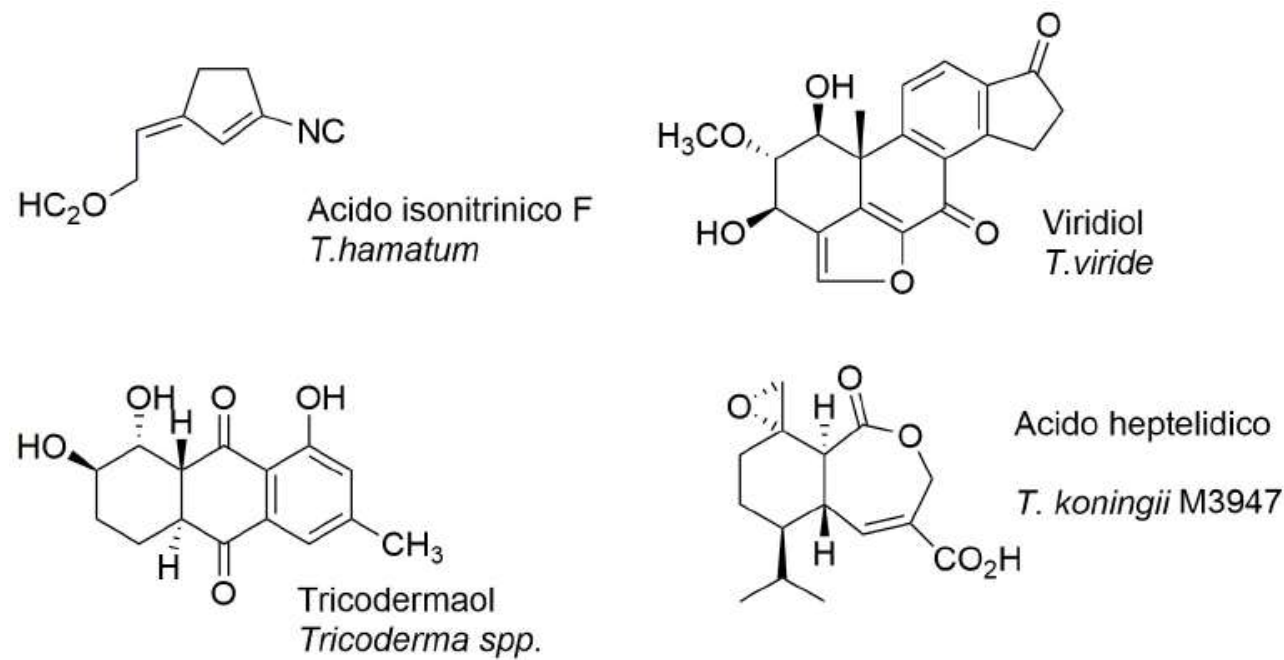

Figura 6. Lactonas aisladas de Trichoderma spp. 
(HR-ESI-MS) y se validó su potencial antibacteriano (Chen et al. 2018, Stoppacher et al. 2010).

\section{Actividad nematicida de metabolitos de Tricho- derma}

Trichoderma es un importante agente de control biológico que produce metabolitos dañinos para los nematodos. Los COVs de la cepa Trichoderma sp. YMF 1.00416 fueron evaluados por su habilidad para eliminar estos helmintos. Las investigaciones químicas de los COVs de esta cepa condujeron al aislamiento y identificación de tres metabolitos: un nuevo compuesto, $1 \beta$-vinilciclopentano- $1 \alpha, 3 \alpha$-diol y dos metabolitos conocidos, 6-pentil-2H-piran-2-ona y 4-(2-hidroxietil) fenol. Ensayos de su acción nematicida mostraron una actividad superior al $>85 \%$ sobre Panagrellus redivivus, Caenorhabditis elegans y Bursaphelenchus xylophilus en $48 \mathrm{~h}$ a una concentración de $200 \mathrm{mg} / \mathrm{L}$. Entre los MS se encuentran COVs a los cuales se atribuye un papel determinante en la comunicación entre microorganismos (Stoppacher et al. 2010).

También se ha comprobado el potencial nematicida de T. asperellum Samuels, Lieckfeldt y Nirenberg mediante el uso de seis cepas seleccionadas previamente por su alta capacidad antagónica, producción de metabolitos (figura 7 ) y adaptación a diversas condiciones ambientales y sustratos como agente de control biológico de Meloidogyne incgnita (Kofoid and White). En los ensayos in vitro se observó el efecto de las cepas sobre juveniles de $M$. incognita. Para el estudio se emplearon plantas de tomate (Solanum lycopersicum L.) que se inocularon, a las $72 \mathrm{~h}$, con 2,5 juveniles de segundo estadio (J2) por gramo de suelo. A los 35 días se evaluaron parámetros como: Índice de Agallamiento (IA), número de huevos del nematodo por sistema radical, altura de planta, diámetro del tallo, longitud de la raíz, número de hojas y masa fresca de raíz. Los extractos de las cepas de $T$. asperellum causaron altos niveles de mortalidad con $90 \%$ de mortalidad a las 24 h (Hernández-Ochandia et al. 2015)
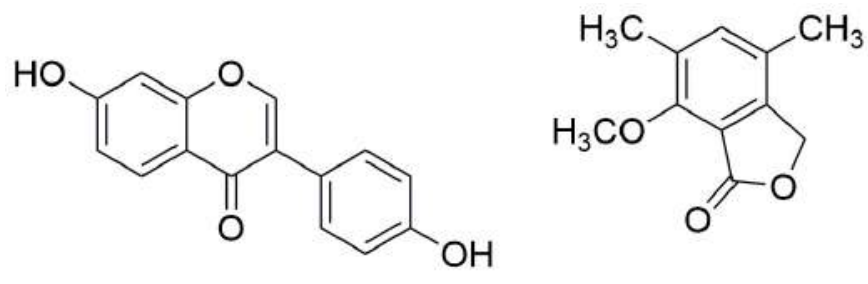

Figura 7. Metabolitos aislados de T. asperellum.

\section{Actividad insecticida de metabolitos de Tricho- derma}

El control de cucarachas ha sido estudiado con cepas de hongos Trichoderma, al determinarse el efecto de las esporas en suspensión y de los MS de T. harzianum contra Periplaneta americana, evidenciado su patogenicidad y efecto insecticida (Abdul-Wahid y Elbanna 2012, Evidente et al. 2008). También se evaluó el efecto larvicida, a las $24 \mathrm{~h}$, de $T$. viride contra larvas de tercer instar del mosquito vector Culex quinquefasciatus, con valores de $\mathrm{CL}_{50}$ de 54,16 $\mathrm{mg} / \mathrm{L}$ (Govindarajan et al. 2005).

\section{CONCLUSIONES}

El género Trichoderma está integrado por un gran número de cepas fúngicas que actúan como agentes de control biológico y cuyas propiedades antagónicas se basan en la activación de mecanismos muy diversos. Debido a sus capacidades versátiles, las comunidades de Trichoderma contribuyen esencialmente a un ambiente saludable pero también representan un recurso de biomoléculas con alto potencial biológico. Estos hongos antagonistas deben estudiarse con mayor profundidad para la identificación de moléculas bioactivas de uso industrial o en formulaciones comerciales de productos para el control biológico de patógenos en plantas. Actualmente se valoran métodos que sean eficientes en la expresión de MS a través de técnicas de fermentación mediante las modificaciones de las condiciones de crecimiento, la variación en los factores reguladores y el uso de nuevas técnicas espectroscópicas, lo que permitirá el descubrimiento de nuevas moléculas, además de la comprensión de su maquinaria de biosíntesis a la luz del conocimiento en la expresión génica y la información adquirida de los genomas de este género. 
La cooperación interdisciplinaria es esencial si queremos entender la función, expresión de la genómica y la proteómica en la producción de MS, además de tener en cuenta otros aspectos como la formulación y estabilidad en almacenaje de productos a base de Trichoderma, como un aspecto importante para obtener un producto final.

Esta revisión presenta una visión general las actividades biológicas presentadas por los MS del género Trichoderma en hongos, bacterias y nematodos, con el fin de encontrar hallazgos y aplicaciones prometedoras en la expresión de cada cepa. Las especies de Trichoderma presentan un efecto positivo en plantas mejorando sus propiedades como la biomasa, rendimiento y calidad, ademas de contribuir al control de plagas y enfermedades en la mayor parte de cultivos agrícolas. Aún quedan muchos MS por descubrir en Trichoderma, ya que este género de hongos incluye múltiples especies que están altamente adaptados a diferentes nichos ecológicos y su diversidad parece ser ilimitada. Esto bien podría conducir al descubrimiento de nuevos compuestos y/o nuevas vías biosintéticas, que a su vez podrían revelar aspectos nuevos e importantes para muchas aplicaciones agrobiotecnológicas.

\section{CONFLICTO DE INTERESES}

Los autores declaran no tener conflictos de intereses.

\section{REFERENCIAS}

Abdul-Wahid OA, Elbanna SM. 2012. Evaluation of the insecticidal activity of Fusarium solani and Trichoderma harzianum against cockroaches Periplaneta americana. African Journal of Microbiology Research, 6(5): 10241032. doi:10.5897/AJMR-11-1300

Andrade R, Ayer WA, Mebe PP. 1992. The metabolites of Trichoderma longibrachiatum. Part 1. Isolation of the metabolites and the structure of trichodimerol. Canadian Journal of Chemistry, 70(10): 2526-253. https://www. nrcresearchpress.com/doi/pdf/10.1139/v92-320

Adekambi S, Adegbola P, Arouna A. 2010. Farmers' perception and agricultural technology adoption. The case of botanical extracts and biopesticides in vegetable production in Benin. Institut National des Recherches Agricoles du Bénin (INRAB), Porto-Novo, Bénin. doi:10.22004/ag.econ.95917

Bae SJ, Mohanta TK, Chung JY, Ryu M, Park G, Shim S, Hong S-B, Seo H, Bae D-W, Bae I, Kim, J-J, Bae
H. 2016. Trichoderma metabolites as biological control agents against Phytophthora pathogens. Biological Control, 92: 128-138. doi:10.1016/j.biocontrol.2015.10.005

Benítez T, Rincón AM, Limón MC, Codon AC. 2004. Biocontrol mechanisms of Trichoderma strains. International Microbiology, 7(4): 249-260. http://www.im.microbios. org/0704/0704249.pdf

Cantrell CL, Dayan FE, Duke SO. 2012. Natural products as sources for new pesticides. Journal of Natural Products, 75(6): 1231-1242. doi:10.1021/np300024u

Chen L, Wu GW, Liu D, Zhuang WY, Yin WB. 2018. Trichodermatides $\mathrm{E}$ and $\mathrm{F}$ from fungus Trichoderma applanatum. Journal of Asian Natural Products Research, 21(7): 659-665. doi:10.1080/10286020.2018.1465051

Contreras-Cornejo HA, Macías-Rodríguez L, del-Val E, Larsen J. 2016. Ecological functions of Trichoderma spp. and their secondary metabolites in the rhizosphere: interactions with plants. FEMS Microbiology Ecology, 92(4): fiw036. doi:10.1093/femsec/fiw036

Dal Bello GM, Mónaco CI, Cháves AR. 1997. Efecto de los metabolitos volátiles de Trichoderma hamatum sobre el crecimiento de hongos fitopatógenos procedentes del suelo. Revista Iberoamericana de Micología, 14: 131-134. http://www.reviberoammicol.com/1997-14/131134.pdf

Elfita E, Munawar M, Muharni M, Sudrajat MA. 2014. Identification of new lactone derivatives isolated from Trichoderma sp., an endophytic fungus of brotowali (Tinaspora crispa). Hayati Journal of Bioscience, 21(1): 15-20. doi: $10.4308 /$ hjb.21.1.15

Eziashi EI, Uma, NU, Adekunle AA, Airede CE. 2006. Effect of metabolites produced by Trichoderma species against Ceratocystis paradoxa in culture medium. African Journal of Biotechnology, 5(9): 703-706. https://www.ajol. info/index.php/ajb/article/view/42775/26344

Evidente A, Ricciardiello G, Andolfi A, Sabatini MA, Ganassi S, Altomare C. 2008. Citrantifidiene and citrantifidiol: bioactive metabolites produced by Trichoderma citrinoviride with potential antifeedant activity toward aphids. Journal of Agricultural and Food Chemistry, 56(10): 3569-3573. doi:10.1021/jf073541h

EPA (United States Environmental Protection Agency). 2016. Integrated Pest Management (IPM) Principles. [fecha de acceso diciembre 2 2016]. https://www.epa.gov/safepestcontrol/ integrated-pest-management-ipm-principles

FAO (Food and Agriculture Organization of the United States). 2019. Agricultura mundial: hacia los años 2015/2030. Rome (Italia): FAO. http://www.fao.org/3/ y3557s/y3557s00.htm

Gakuya D, Itoga S, Mbaria J, Muthee J, Musau J. 2013. Ethnobotanical survey of biopesticides and other medicinal plants traditionally used in Meru central district of Kenya. Journal of Ethnopharmacology, 145(2): 547-553. doi:10.1016/j.jep.2012.11.028

García R, Riera R, Zambrano C, Gutiérrez L. 2006. Desarrollo de un fungicida biológico a base de una cepa del hongo Trichoderma harzianum proveniente de la región andina venezolana. Fitosanidad, 10(2): 115-121. https://www.redalyc.org/articulo.oa?id=209116102005

Gavrilescu M, Chisti Y. 2005. Biotechnology a sustainable al- 
ternative for chemical industry. Biotechnology Advances, 23 (7-8): 471-499. doi:10.1016/j.biotechadv.2005.03.004

Ghisalberti EL, Rowland CY. 1993. Antifungal metabolites from Trichoderma harzianum. Journal of Natural Products, 56(10): 1799-1804. doi:10.1021/np50100a020

Govindarajan M, Jebanesan A, Reetha D. 2005. Larvicidal effect of extracellular secondary metabolites of different fungi against the mosquito, Culex quinquefasciatus Say. Tropical Biomedicine, 22(1): 1-3. http://www.msptm. org/files/1_-_3_Larvicidal_effect_of_extracellular.pdf

Gupta VG, Schmoll M, Herrera-Estrella A, Upadhyay RS, Druzhinina I, Tuohy M, editors. 2014. Biotechnology and biology of Trichoderma. Newnes. Amsterdam: Elsevier. doi:10.1016/C2012-0-00434-6

Harman GE, Kubicek CP, editores. 2014. Trichoderma and Gliocladium. Basic biology, taxonomy and genetics. Vol. 1. Boca Raton (USA): CRC Press. doi: $10.1201 / 9781482295320$

Hernández-Ochandía D, Rodríguez MG, Peteira B, Miranda I, Arias Y, Martínez B. 2015. Efecto de cepas de Trichoderma asperellum Samuels, Lieckfeldt y Nirenberg sobre el desarrollo del tomate y Meloidogyne incognita (Kofoid y White) Chitwood. Revista de Protección Vegetal, 30(2): 139-147. http://scielo.sld.cu/pdf/rpv/v30n2/ rpv08215.pdt

Hoyos-Carvajal L. 2012. Enfermedades de plantas: control biológico. Bogotá: ECOE ediciones.

Infante D, Martínez B, González N, Reyes Y. 2009. Mecanismos de acción de Trichoderma frente a hongos fitopatógenos. Revista de Protección Vegetal, 24(1): 14-21. http://scielo.sld.cu/scielo.php?script=sciarttext\&pid=S1010-27522009000100002

International Subcommission on Trichoderma and Hypocrea Taxonomy. 2004. International Commission for the Taxonomy of Fungi (ICTF), International Union of Microbiological Societies (IUMS, Mycology Division). [fecha de acceso septiembre, 2019]. http://www.isth.info/

Keswani C, Mishra S, Sarma BK, Singh SP, Singh HB. 2014. Unraveling the efficient applications of secondary metabolites of various Trichoderma spp. Applied Microbiology and Biotechnology, 98(2): 533-544. doi:10.1007/s00253III.3-5:344-5

Kong Z, Jing R, Wu Y, Guo Y, Geng Y, Ji J, Zheng C. 2018. Trichodermadiones A and B from the solid culture of Trichoderma atroviride S361, an endophytic fungus in Cephalotaxus fortunei. Fitoterapia, 127: 362-366. doi:10.1016/j.fitote.2018.04.004

León DCS, Cortés ACP, Sarmiento NCM. 2017. Evaluación de la actividad fungicida e identificación de compuestos orgánicos volátiles liberados por Trichoderma viride. Revista Colombiana de Biotecnología, 19(1): 63-70. doi:10.15446/rev.colomb.biote.v19n1.65969

Lorito M. 2006. La biología molecular de las interacciones entre Trichoderma, hongos fitopatógenos y plantas: oportunidades para desarrollar nuevos métodos de control de enfermedades. Fitosanidad 10(2): 139-140.

Marques E, Martins I, Mello SCMD. 2018. Antifungal potential of crude extracts of Trichoderma spp. Biota Neotropica, 18(1): e20170418 10.1590/1676-0611-bn-2017-0418

Martínez B, Infante D, Reyes Y. 2013. Trichoderma spp. y su función en el control de plagas en los cultivos. Revista de Protección Vegetal, 28(1): 1-11. http://scielo.sld.cu/pdf/ rpv/v28n1/rpv01113.pdt

Mona SA, Hashem A, Abd Allah EF, Alqarawi AA, Soliman DWK, Wirth S, Egamberdieva D. 2017. Increased resistance of drought by Trichoderma harzianum fungal treatment correlates with increased secondary metabolites and proline content. Journal of Integrative Agriculture, 16(8): 1751-1757. http://www.chinaagrisci.com/Jwk zgnykxen/EN/10.1016/S2095-3119(17)61695-2

Mukherjee PK, Horwitz BA, Kenerley CM. 2012. Secondary metabolism in Trichoderma-a genomic perspective. Microbiology, 158(1): 35-45. doi:10.1099/mic.0.053629-0

Okuda T, Fujiwara A, and Fujiwara M. 1982. Correlation between species of Trichoderma and production patterns of isonitrile antibiotics. Agricultural and Biological Chemistry, 46(7): 1811-1822. doi:10.1080/00021369.1982.10865345

Pascale A, Vinale F, Manganiello G, Nigro M, Lanzuise S, Ruocco M, Lorito M. 2017. Trichoderma and its secondary metabolites improve yield and quality of grapes. Crop Protection, 92(1): 176-181. doi:10.1016/j.cropro.2016.11.010

Pathak J, Pandey A, Singh SP, Sinha RP. 2017. World Agriculture and Impact of Biotechnology. En: Dubey SK, Pandey A, Sangwan RS. Current Developments in Biotechnology and Bioengineering. Amsterdam: Elsevier. p.1-22. doi:10.1016/B978-0-444-63661-4.00001-3

Puño R, Terrazas E, Alvares T, Giménez A, Mendoza L, Smeltekop H, Loza-Murguia M. 2011. Evaluación de la capacidad biocontroladora de metabólicos de Trichoderma inhamatum Bol12 QD sobre cepas nativas de Phytophthora infestans in vitro. Journal of the Selva Andina Research Society, 2(1): 26-33. http://www.scielo.org.bo/ pdf/jsars/v2n1/a04.pdf

Reino JL, Guerrero RF, Hernández-Galán R, Collado IG. 2007. Secondary metabolites from species of the biocontrol agent Trichoderma. Phytochemistry Reviews, 7(1): 89123. doi:10.1007/s11101-006-9032-2

Ruane J, Sonnino A. 2011. Agricultural biotechnologies in developing countries and their possible contribution to food security. Journal of Biotechnology, 156(4): 356-363. doi:10.1016/j.jbiotec.2011.06.013

Saeed MF, Shaheen M, Ahmada I, Zakir A, Nadeema M, Chishti AA, Shahid M, Bakhsh K, Damalas CA. 2017. Pesticide exposure in the local community of Vehari District in Pakistan: An assessment of knowledge and residues in human blood Author links open overlay panel. Science of The Total Environment. 587-588: 137-144. doi:10.1016/j.scitotenv.2017.02.086

Saleh RM, Kabli SA, AlGarni SM, AlGhamdi MA, AbdelAty AM, Mohamed SA. 2018. Solidstate fermentation by Trichoderma viride for enhancing phenolic content, antioxidant and antimicrobial activities in ginger. Letters in Applied Microbiology, 67(2):161-167. doi:10.1111/lam.13003

Saravanakumar K, Chelliah R, Ramakrishnan SR, Kathiresan K, Oh DH, Wang MH. 2018. Antibacterial, and antioxidant potentials of non-cytotoxic extract of Trichoderma atroviride. Microbial Pathogenesis, 115: 338-342. 
doi:10.1016/j.micpath.2017.12.081

Schulz B, Boyle C, Draeger S, Römmert AK, Krohn K. 2002. Endophytic fungi: a source of novel biologically active secondary metabolites. Mycological Research, 106(9): 9961004. doi:10.1017/S0953756202006342

Sivasithamparam K, Ghisalberti EL. 1998. Secondary Metabolism in Trichoderma and Gliocladium. En: Kubicek CP, Harman GE, editores. Trichoderma and Gliocladium. Vol. 1. Londres: Taylor and Francis Ltd. p. 139-191.

Smirnova IP, Karimova EV, Shneider YA. 2017. Antibacterial Activity of L-Lysine- $\alpha$-Oxidase from the Trichoderma. Bulletin of Experimental Biology and Medicine, 163(6): 777-779. doi:10.1007/s10517-017-3901-0

Stoppacher N, Kluger B, Zeilinger S, Krska R, Schuhmacher R. 2010. Identification and profiling of volatile metabolites of the biocontrol fungus Trichoderma atroviride by HS-SPME-GC-MS. Journal of Microbiological Methods, 81(2): 187-193. doi:10.1016/j.mimet.2010.03.011

Sun J, Pei Y, Li E, Li W, Hyde K.D, Yin WB, Liu X. 2016. A new species of Trichoderma hypoxylon harbours abundant secondary metabolites. Scientific Reports, 6: 37369. doi:10.1038/srep37369

Verma, M, Brar SK, Tyagi RD, Surampalli RY, Valero JR. 2007. Antagonistic fungi, Trichoderma spp.: panoply of biological control. Biochemical Engineering Journal, 37(1): 1-20. doi:10.1016/j.bej.2007.05.012

Vinale F, Sivasithamparam K, Ghisalberti EL, Marra R, Barbetti MJ, Li H, Lorito M. 2008. A novel role for Trichoderma secondary metabolites in the interactions with plants. Physiological and Molecular Plant Pathology, 72(1-3): 80-86. doi:10.1016/j.pmpp.2008.05.005

Vinale F, Sivasithamparam K, Ghisalberti EL, Ruocco M, Wood S, Lorito M. 2012. Trichoderma secondary metabolites that affect plant metabolism. Natural Product Communications, 7(11): 1545-1550. https://journals.sagepub. com/doi/pdf/10.1177/1934578X1200701133

Yang Z, Yu Z, Lei L, Xia Z, Shao L, Zhang K, Li G. 2012. Nematicidal effect of volatiles produced by Trichoderma sp. Journal of Asia-Pacific Entomology, 15(4): 647-650. doi:10.1163/15685411-00002920

Zeilinger S, Gruber S, Bansal R, Mukherjee PK. 2016. Secondary metabolism in Trichoderma-Chemistry meets genomics. Fungal Biology Reviews, 30(2): 74-90. doi:10.1016/j.fbr.2016.05.001

Zhang M, Liu JM, Zhao J-L, Li N, Chen R-D, Xie KB, Zhang W-J, Feng KP, Yan Z, Wang N, Dai JG. 2016. Two new diterpenoids from the endophytic fungus Trichoderma sp. Xy24 isolated from mangrove plant Xylocarpus granatum. Chinese Chemical Letters, 27(6): 957-960. doi:10.1016/j.cclet.2016.02.008

Zhou P, Wu Z, Tan D, Yang J, Zhou Q, Zeng F, Luo Z. 2017. Atrichodermones A-C, three new secondary metabolites from the solid culture of an endophytic fungal strain, Trichoderma atroviride. Fitoterapia, 123: 18-22. doi:10.1016/j.fitote.2017.09.012 